Schmerz 2010 $24: 313-314$

DOI 10.1007/s00482-010-0947-6

Online publiziert: 29. Juli 2010

(c) Deutsche Gesellschaft zum Studium des Schmerzes. Published by Springer

Medizin Verlag - all rights reserved 2010

\author{
L. Radbruch ${ }^{1}$. F. Nauck ${ }^{2}$ \\ ${ }^{1}$ Klinik für Palliativmedizin, RWTH Aachen \\ ${ }^{2}$ Abteilung Palliativmedizin, Zentrum Anaesthesiologie, Rettungs- \\ und Intensivmedizin, Georg-August-Universität Göttingen
}

\section{Sedieren oder nicht sedieren - das ist hier die Frage}

\section{Palliative Sedierung zwischen Standard und Flexibilität}

Vor mittlerweile mehr als 1o Jahren saßen die Autoren dieses Editorials in einem Expertenworkshop in San Raffaele in Südfrankreich. Das Thema war die terminale Sedierung. Der Workshop war von der European Association for Palliative Care (EAPC) einberufen worden mit dem Auftrag, europäische Leitlinien mit einem Standard für Indikation, Vorbereitung und Durchführung dieser Intervention $\mathrm{zu}$ formulieren. Wir waren $\mathrm{zu}$ versichtlich, dass uns dies gelingen würde, hatte doch die EAPC mit anderen Arbeitsgruppen bei ähnlichen Treffen bereits erfolgreich Empfehlungen zur Schmerzerfassung [4], zum Einsatz von Opioiden [7], zum Durchbruchschmerz [8], zur Ernährung [3] und zur Behandlung von Depressionen [13] veröffentlicht. Auch hatten wir ja schon in früheren Konferenzen und Workshops unsere Standpunkte diskutiert und Meinungen ausgetauscht.

In dem 2-tägigen Workshop konnte jedoch nur Einigkeit zur Terminologie erzielt werden: Zukünftig sollte von palliativer und nicht von terminaler Sedierung gesprochen werden. Damit sollte klargestellt werden, dass diese Intervention nicht zwingend zum Tode hinführt, sondern eine wertvolle palliativmedizinische Intervention darstellt.

In der Folge haben wir an einer ganzen Reihe von Workshops und Konferenzen teilgenommen, die wiederum versucht haben, Leitlinien zur palliativen Sedierung zu erstellen, mit wenig Erfolg. Erst 2007 konnte ein internationaler Konsens veröffentlicht werden $[5,10]$, der allerdings nur wenig beachtet wurde und dessen Empfehlungen weiterhin kontrovers diskutiert wurden.

Dem Vorstand der EAPC war jedoch weiterhin gegenwärtig, dass die Diskussion um die palliative Sedierung nicht abbricht. Auf der einen Seite haben Kritiker der Methode die palliative Sedierung in die Nähe der aktiven Sterbehilfe gerückt $[2,6]$. Tatsächlich wurde auch von einem Missbrauch der Methode berichtet, in dem die Sedierung mit dem Ziel der Lebensverkürzung eingesetzt wurde [12]. Auf der anderen Seite wird von zunehmend häufigeren Anforderungen von Patienten und Angehörigen nach einer solchen Sedierung am Lebensende und in der Folge auch von einem Anstieg der Häufigkeiten in deutschen palliativmedizinischen Einrichtungen berichtet [9].

Die EAPC hat deshalb Nathan Cherny mit der Erstellung aktueller Empfehlungen zur palliativen Sedierung beauftragt. Er fand gemeinsam mit anderen Experten eine Lösung, um die unterschiedlichen Positionen in der Diskussion zu vereinen, indem er ein Rahmenwerk statt starrer Leitlinien anbot. In diesem Rah- menwerk werden die Bereiche und Themen benannt, die beachtet werden müssen. Wie diese Bereiche geregelt werden, wird aber den einzelnen Einrichtungen, Gesellschaften oder Ländern überlassen, die aufgefordert werden, aus diesem Rahmenwerk eigene Leitlinien zu erarbeiten, nachdem die ethischen und organisatorischen Präferenzen und Wertungen in den einzelnen Bereichen diskutiert worden sind.

Die Kombination des festen Rahmens, der klar die Grundpositionen vorgibt, mit den individuellen Anpassungen an die Besonderheiten der regionalen oder nationalen Situation führt aus unserer Sicht zu einer optimalen Kombination von Standardisierung und Flexibilität. Mit dieser Kombination ließ sich zum ersten Mal in der EAPC ein breiter Konsens finden. Die Übersetzung des Rahmenwerkes durch die nationalen Fachgesellschaften ist nun der nächste Schritt in der Implementierung. Die deutsche Übersetzung des Rahmenwerkes wurde gerade in der Zeitschrift für Palliativmedizin veröffentlicht [1] und wird in diesem Heft nachgedruckt.

Von der Arbeitsgemeinschaft Ethik am Lebensende in der Akademie für Ethik in der Medizin werden gerade Empfehlungen vorgelegt zur Sedierung am Lebensende [11]. Allerdings weichen die Empfehlungen von dem europäischen Rah- 
menwerk in einigen zentralen Punkten ab. So wird die Sedierung in eine oberflächliche (Stufe 1) und eine tiefe (Stufe 2) Form unterschieden, wobei Stufe 1 als reversibel und Stufe 2 als irreversibel dargestellt wird. Dies widerspricht aber der klinischen Praxis zumindest in unseren Abteilungen. Wir haben in Einzelfällen durchaus mit Patienten abgestimmt, dass nach einer festgesetzten Zeit die „tie$\mathrm{fe}$ " Sedierung wieder gestoppt wird und vielleicht später wieder begonnen werden kann. Bei anderen Patienten wurde die Sedierung aus anderen Gründen unterbrochen. Es ist doch geradezu ein Merkmal der palliativen Sedierung, dass sie jederzeit reversibel ist und sein muss. Das Rahmenwerk der EAPC bezieht die Sedierung nicht nur auf das Lebensende, sondern stellt auch Indikationen wie die wiederholte kurzzeitige Sedierung z. B. bei besonders schmerzhaftem Verbandswechsel vor.

Darüber hinaus legen die Empfehlungen der Arbeitsgemeinschaft fest, dass bei der tiefen Sedierung begleitende Therapien, einschließlich Ernährung und $\mathrm{Hy}$ drierung, nicht indiziert seien und deshalb auf sie verzichtet werden solle, da sie lediglich den Sterbeprozess verlängern. Demgegenüber legt das Rahmenwerk der EAPC fest, dass die Entscheidung für Fortsetzung, Abbruch oder Verzicht von Flüssigkeits- oder Nahrungszufuhr unabhängig von der Entscheidung zur palliativen Sedierung zu treffen ist. Dies steht vor dem Hintergrund der internationalen Diskussion, in der die feste Kombination von Sedierung mit dem Abbruch von Flüssigkeits- und Nahrungszufuhr von Kritikern als Argument für die Nähe zur Euthanasie genannt wird, da die Entscheidung zur Sedierung durch den Verzicht auf Hydration nun zum Tode führe.

Die Diskussion um die palliative Sedierung ist also bei Weitem noch nicht abgeschlossen. Es scheint aber auch wichtig, dass in diesem Grenzbereich der Medizin Standards und Empfehlungen kritisch hinterfragt werden und strittige Punkte rechtzeitig erkannt werden. Es wäre äußerst bedenklich, wenn die palliative Sedierung zu einer standardisierten Routine, vielleicht sogar ausschließlich auf Nachfrage des Patienten, der Angehörigen oder der Behandler werden würde und die Behandler nicht mehr für jeden einzelnen Patienten die medizinische Indikation stellen und die ethische Diskussion annehmen würden. Die palliative Sedierung ist eine wertvolle Therapieoption für die Patienten, bei denen eine Linderung von Symptomen und Leid mit anderen Mitteln nicht möglich ist, aber sie ist auch eine ethische Herausforderung in jedem einzelnen Fall, der wir uns stellen müssen.
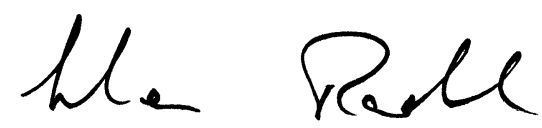

L. Radbruch

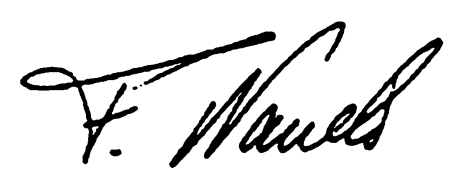

F. Nauck

\section{Korrespondenzadresse}

Prof. Dr. L. Radbruch

Klinik für Palliativmedizin, RWTH Aachen

Pauwelssstr. 30, 52074 Aachen

LRadbruch@ukaachen.de

\section{Literatur}

1. Alt-Epping B, Sitte T, Nauck F, Radbruch L (2010) Sedierung in der Palliativmedizin - Leitlinie für den Einsatz sedierender Maßnahmen in der Palliativversorgung. Z Palliativmed 11:112-122

2. Billings JA, Block SD (1996) Slow euthanasia. J Palliat Care 12(4):21-30

3. Bozzetti F, Amadori D, Bruera E et al (1996) Guidelines on artificial nutrition versus hydration in terminal cancer patients. European Association for Palliative Care. Nutrition 12(3):163-167

4. Caraceni A, Cherny N, Fainsinger R et al (2002) Pain measurement tools and methods in clinical research in palliative care. Recommendations of an expert working group of the European Association of Palliative Care. J Pain Symptom Manage 23(3):239-255

5. de Graeff A, Dean M (2007) Palliative sedation therapy in the last weeks of life: a literature review and recommendations for standards. J Palliat Med 10(1):67-85

6. Gevers JK (2006) Terminal sedation: between pain relief, withholding treatment and euthanasia. Med Law 25(4):747-751

7. Hanks GW, Conno F, Cherny N et al (2001) Morphine and alternative opioids in cancer pain: the EAPC recommendations. Br J Cancer 84(5):587593

8. Mercadante S, Radbruch L, Caraceni A et al (2002) Episodic (breakthrough) pain: consensus conference of an expert working group of the European Association for Palliative Care. Cancer 94(3):832-839
9. Muller-Busch HC, Andres I, Jehser T (2003) Sedation in palliative care - a critical analysis of 7 years experience. BMC Palliat Care 2(1):2

10. Muller-Busch HC, Radbruch L, Strasser F, Voltz R (2006) Empfehlungen zur palliativen Sedierung. Dtsch Med Wochenschr 131(48): 2733-2736

11. Neitzke G, Oehmichen F, Schliep HJ, Wördehoff D (2010) Sedierung am Lebensende: Empfehlungen der AG Ethik am Lebensende in der Akademie für Ethik in der Medizin (AEM). Ethik in der Medizin (online first)

12. Rietjens $J A$, van der Heide $A$, Vrakking AM et al (2004) Physician reports of terminal sedation without hydration or nutrition for patients nearing death in the Netherlands. Ann Intern Med 141(3):178-185

13. Stiefel F, Die Trill M, Berney A et al (2001) Depression in palliative care: a pragmatic report from the Expert Working Group of the European Association for Palliative Care. Supp Care Cancer 9:477-488 